\title{
¿Mejora la CIE-11 los propósitos epidemiológicos y nosológicos de los Trastornos mentales, del comportamiento y del desarrollo?
}

\section{Does ICD-11 improve the epidemiological and nosological purposes of mental, behavioral and developmental disorders?}

\author{
María Teresa Bobes Bascarán*, Gerardo Flórez**, Pedro Seijo***, Julio Bobes García**** \\ *Servicio de Salud del Principado de Asturias (SESPA). Departamento de Psicología. Universidad de Oviedo-ISPA-INEUROPA- \\ CIBERSAM \\ **Unidad de Conductas Adictivas, Complejo Hospitalario Universitario de Ourense, España. CIBERSAM \\ *** Centro de Tratamiento Ambulatorio de Adicciones Villamartín, Diputación de Cádiz. Psiquiatra Consultor Centros \\ Penitenciarios Sevilla I y II. España. \\ ****Área de Psiquiatría, Departamento de Medicina. Universidad de Oviedo- ISPA-INEUROPA-CIBERSAM
}

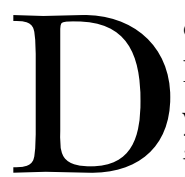
esde la primera Clasificación Internacional de Enfermedades (CIE) en 1893, se han revisado y publicado una serie de ediciones para reflejar los avances en salud y ciencia médica. A la Organización Mundial de la Salud (OMS) se le confió la CIE en 1948 y publicó la sexta version que incorporaba la morbilidad por primera vez. El Reglamento de la Nomenclatura de la OMS estipula que los Estados miembros utilicen la revisión más reciente de la CIE para las estadísticas de mortalidad y morbilidad. Han pasado ya 29 años desde que fuera aprobada por la $43^{\text {a }}$ Asamblea Mundial de la Salud la CIE-10 y por tanto, tras la 72a Asamblea Mundial de la Salud que se acaba de celebrar en Ginebra (Suiza) del 20 al 28 de mayo de 2019, los Estados miembros comenzarán a presentar informes sobre estadísticas de salud basadas en el nuevo sistema el 1 de enero de 2022 (Organización Mundial de la Salud, 2019). De manera subsiguiente está previsto que, una vez aprobada por dicha Asamblea, el Departamento de Salud Mental y Abuso de Sustancias de la OMS publique las Descripciones Clínicas y Pautas de Diagnóstico (DCPD) para los Trastornos Mentales, del Comportamiento y del Neurodesarrollo de la CIE-11. Estas DCPD son el resultado de un trabajo sistemático realizado durante la última década, basado en los principios de utilidad clínica y aplicabilidad global, y supone el proceso de revisión de mayor alcance internacional, plurilingüe, multidisciplinario y participativo jamás implementado para una clasificación de trastornos mentales (Reed et al., 2019). Entre las novedades que aporta la CIE-11 destacan la inclusión de información coherente y sistemáticamente detallada, la adopción de un enfoque a lo largo del ciclo vital (lifespan) y la aproximación cultural para cada trastorno. Se ha incorporado una perspectiva dimensional a la clasificación, específicamente para los trastornos de personalidad y los trastornos psicóticos primarios, de tal forma que son consistentes con la evidencia actual, son más compatibles con los enfoques basados en la recuperación, eliminan la comorbilidad artificial y captan los cambios longitudinales de manera más efectiva (Reed et al., 2019; Robles Garcia y Ayuso-Mateos, 2019). En los estudios realizados por los diferentes grupos de trabajo, se concluye que dichas pautas se percibieron como fáciles de usar, correspondiendo con precisión a las presentaciones de enfermedad de los pacientes (es decir, la bondad del ajuste), de forma clara y comprensible pero con un nivel de detalle apropiado (Ayuso Mateos, 2018). Así mismo, parece que los DCPD requieren incluso menos tiempo que la práctica habitual de los médicos, y aportan información útil a la hora de orientar el trastorno frente a la normalidad (Reed, Keeley et al., 2018; Stein y Reed, 2019).

\section{Los cimientos de la CIE-11}

Tanto la neurociencia clínica como la salud mental global han enfatizado que el diagnóstico psiquiátrico no debe conceptualizarse en términos de categorías esencialistas, los mecanismos biológicos conducen a un espectro de síntomas, y los servicios de salud deben responder a las

Recibido: Abril 2019; Aceptado: Junio 2019.

Enviar correspondencia a:

María Teresa Bobes Bascarán. Centro de Salud Mental II- La Corredoria, Avd. Alfredo Blanco s/n 33011, Oviedo (España)

E-mail: mteresa.bobes@sespa.es 
diversas etapas de la enfermedad mental al proporcionar niveles de atención escalonados. Los marcos como los Criterios de Dominio de Investigación (RDoC) ponen énfasis específico en estos mecanismos biológicos subyacentes y en los espectros de síntomas que sustentan. En el contexto de estos debates y controversias, el capítulo de trastornos mentales, del comportamiento y del desarrollo neurológico de la CIE-11 representa un importante paso adelante para el campo de la salud mental global en general, y para los servicios e investigaciones en entornos socioeconómicos bajos y medios tal y como propone el Plan de Acción de Salud Mental 2013-2020 (Organización Mundial de la Salud, 2013). Esto se debe a que esta nueva edición se ha basado en las fortalezas clave incorporadas en el DSM-5 y en los RDoC (Stein y Reed, 2019), es decir, se enfatiza la toma de decisiones nosológicas respaldada por la evidencia y la visión traslacional de la investigación.

Dicho esto, se ha de resaltar el importante esfuerzo de la CIE-11 para superar las limitaciones clave del DSM y de los RDoC. Los productos del DSM son caros y generan importantes beneficios para la Asociación de Psiquiatría Americana (APA). En contraste, los productos de la CIE están disponibles gratuitamente en todo el mundo lo que aumenta la probabilidad de que se utilicen en una amplia gama de entornos para mejorar el diagnóstico y el tratamiento. Por otra parte, las construcciones de los RDoC son complejas y se adaptan en mayor medida a entornos académicos de investigación intensiva. En contraste, las construcciones de la CIE son fáciles de usar y resultan más adecuados para su adopción por no especialistas en entornos de atención primaria de todo el mundo (Reed y Ayuso-Mateos, 2011). Finalmente, la CIE-11 ha mantenido su propia identidad y visión únicas. Los críticos de la nosología psiquiátrica pueden argumentar que la existencia misma de diferentes enfoques de clasificación habla de fallas en el campo. Esta crítica no comprende que la nosología psiquiátrica no se trata únicamente de identificar categorías esencialistas sino que más bien, la clasificación pretende ser apta para cada propósito (Keeley et al., 2018). En un entorno de investigación altamente especializado es útil emplear un sistema de clasificación que enfatice criterios de diagnóstico rigurosos y subtipos de trastorno específicos. Sin embargo, en una amplia gama de entornos globales, es más probable que un sistema que brinde una orientación más flexible y algo menos granular sea percibida como aceptable y factible y por tanto sea más fácil que se adopte y que conduzca a mejores resultados para el paciente (Medina-Mora et al., 2019; Peterson et al., 2019). De cualquier manera, el énfasis fundamental en que esta clasificación sea de utilidad clínica en diferentes países asegura que estemos antes una herramienta clave para la salud mental a nivel global (Organización Mundial de la Salud, 2013; Reed, Keeley et al., 2018; Reed et al., 2018).

\section{Los trastornos por uso de sustancias como problema de salud pública}

El enfoque propuesto por la CIE-11 para la clasificación de trastornos debidos al uso de sustancias se basa en la aproximación al concepto de salud pública como marco de referencia. Desde la perspectiva de la salud pública se busca prevenir enfermedades, prolongar la vida y promover la salud a través de esfuerzos organizados y decisiones informadas de la sociedad, las organizaciones, las comunidades públicas y privadas y las personas. Este hecho se ve reflejado en las políticas internacionales de drogas que adoptan el bienestar de las personas y las comunidades en lugar de las incautaciones de drogas como los principales indicadores de éxito dentro del Plan de acción europeo en materia de lucha contra la droga 2017-2020 (Consejo de Europa, 2017). Esta nueva perspectiva choca con la que había hace unas décadas, que concebía las adicciones como un problema social o un defecto de carácter e incluso con el hecho de que actualmente, el consumo de drogas aún es punible con encarcelamiento en algunos países, y los centros de detención obligatoria de drogas siguen siendo proveedores importantes de "tratamiento" no voluntario en muchas partes del mundo, a pesar de la evidencia de su ineficacia (Wegman et al., 2017). Al mismo tiempo la diversificación de las sustancias psicoactivas y los cambios en sus vías de administración y los contextos de su uso presentan nuevos desafíos para los gobiernos y los sistemas de salud (Dolengevich-Segal, Rodriguez Salgado, Gomez-Arnau Ramirez y Sanchez-Mateos, 2015; Dolengevich-Segal, Rodriguez Salgado, Ballesteros-López y Molina-Prado, 2017). El enfoque de salud pública reconoce el consumo de sustancias y los trastornos por el consumo de sustancias como un espectro de comportamientos y afecciones de salud que requieren diferentes enfoques, servicios y recursos para lograr los objetivos de salud pública. Es fundamental que la clasificación de los trastornos por consumo de sustancias cubra diferentes fases y patrones de consumo a través de un conjunto de categorías de diagnóstico que se organicen en un continuo que refleje las etapas y la gravedad del trastorno. Por lo tanto, la clasificación de los trastornos debidos al consumo de sustancias en la CIE-11 pretende que sea utilizada por una amplia gama de profesionales de la salud para cubrir todo el espectro de problemas relacionados para lograr un impacto en la salud de la población (Poznyak, Reed y Medina-Mora, 2018).

\section{Innovaciones respecto a la nosología de las adicciones}

La estructura y las definiciones propuestas para las categorías de diagnóstico de la CIE-11 están disponibles en la plataforma de la OMS (Organización Mundial de la Salud, 2019). Los Trastornos por consumo de sustancias y comportamientos adictivos constituyen una agrupación 
de trastornos que se desarrollan como resultado del uso de sustancias psicoactivas, incluidos los medicamentos, y trastornos debidos a conductas adictivas que se desarrollan como resultado de comportamientos específicos de recompensa y refuerzo repetitivos (Heinz, Daedelow, Wackerhagen y Di Chiara, 2019). Al igual que en la CIE-10, se ha de identificar primero la sustancia y luego calificar el síndrome clínico apropiado.

Los diagnósticos primarios mutuamente excluyentes son:

- Episodio único de consumo nocivo de sustancias

- Patrón de consumo nocivo de sustancias

- Dependencia de sustancias

Además, los siguientes diagnósticos pueden ser motivo de prestación de servicios de salud:

- Intoxicación por sustancias

- Abstinencia de sustancias

Los principales cambios propuestos se señalan en la Tabla 1 e incluyen: (1) un rango actualizado y ampliado de tipos de sustancias psicoactivas; (2) mayor especificación de los diferentes patrones perjudiciales de consumo de sustancias, que pueden ser continuos o episódicos y recurrentes; (3) una nueva categoría para denotar episodios únicos de consumo nocivo; (4) una categoría que describe el consumo nocivo de sustancias, que aunque no se considera un trastorno, se ha incluido en el capítulo de la CIE-11 sobre "Factores que influyen en el estado de salud o el contacto con los servicios de salud"; y (5) simplificación de las guías de diagnóstico para la dependencia de sustancias. Además tanto la intoxicación como la abstinencia de sustancias se pueden diagnosticar junto con los síndromes clínicos primarios, o de manera independiente como motivo para la prestación de servicios de salud cuando se desconoce el patrón de consumo o si existe una dependencia a sustancias. El trastorno amnésico inducido por sustancias y la demencia primaria inducida por sustancias del capítulo Trastornos neurocognitivos también se incluyen en los trastornos por consumo de sustancias. El consumo perjudicial se incluye en el epígrafe de "Problemas asociados con los comportamientos de salud" del capítulo de "Factores que influyen en el estado de salud o el contacto con los servicios de salud". Otra novedad a reseñar es que se puede codificar "episodio único de consumo nocivo" y "patrón de consumo nocivo" cuando el daño surge del consumo de sustancias no psicoactivas como por ejemplo, el abuso de esteroides (Gonzalez-Marti, Fernandez-Bustos, Contreras Jordan y Sokolova, 2018).

\section{Clasificación actualizada y ampliada de clases de sustancias}

En este capítulo, tal como ya hiciera el DSM-5 (Asociación Americana de Psiquiatría, 2014), se presentan los trastornos mentales y del comportamiento que se desarrollan como resultado bien del consumo de sustancias predominantemente psicoactivas, incluidos los medicamentos, bien de comportamientos específicos de recompensa y refuerzo repetitivos. En este sentido, es importante señalar que se da una menor importancia al desarrollo de la tolerancia y los síntomas de abstinencia, ya que pueden ocurrir con cualquier agente farmacológicamente activo y su presencia no es un criterio suficiente para el diagnóstico clínico de un trastorno adictivo. Por el contrario, son criterios clave el craving o ansia de consumir drogas o de realizar el compor-

Tabla 1. Diferencias fundamentales de la adicción a sustancias entre la CIE-10 y la CIE-11

\begin{tabular}{|c|c|c|}
\hline $\begin{array}{l}\text { Trastornos por consumo de sustancias } \\
\qquad(\mathrm{CIE}-11)\end{array}$ & $\begin{array}{l}\text { Trastornos mentales y del comportamiento debido } \\
\text { al consumo de sustancias psicoactivas (CIE-10) }\end{array}$ & Notas \\
\hline Intoxicación por sustancias & $\begin{array}{l}\text { F1x.0 Intoxicación aguda } \\
\text { (x se refiere a la sustancia o tipo de sustancia) }\end{array}$ & $\begin{array}{l}\text { Calificadores omitidos en la CIE-11 } \\
\text { Delirium inducido por sustancias puede } \\
\text { codificarse junto con Intoxicación aguda }\end{array}$ \\
\hline $\begin{array}{l}\text { Episodio único de consumo perjudicial de } \\
\text { sustancias }\end{array}$ & F1x.1 Consumo perjudicial & $\begin{array}{l}\text { Calificadores de patrón prejudicial incluyen } \\
\text { episódico y continuo }\end{array}$ \\
\hline Dependencia de sustancias & F1x.2 Síndrome de dependencia & Calificadores simplificados \\
\hline Abstinencia de sustancias & $\begin{array}{l}\text { F1x.3 Estado de abstinencia } \\
\text { F1x.4 Estado de abstinencia con delirium }\end{array}$ & $\begin{array}{l}\text { Delirium inducido por sustancias puede } \\
\text { codificarse junto con síndrome de abstinencia }\end{array}$ \\
\hline Trastorno psicótico inducido por sustancias & F1x.5 Trastorno psicótico & \\
\hline $\begin{array}{l}\text { Trastorno amnésico inducido por } \\
\text { sustancias* }\end{array}$ & F1x.6 Síndrome amnésico & $\begin{array}{l}\text { *Incorporado primariamente a Trastornos } \\
\text { neurocognitivos de la } \mathrm{CIE}-11\end{array}$ \\
\hline $\begin{array}{l}\text { Demencia inducida por sustancias*, } \\
\text { Trastorno perceptivo persistente inducido } \\
\text { por alucinógenos }\end{array}$ & F1x.7 Trastorno psicótico residual y de inicio tardío & $\begin{array}{l}\text { *Incorporado primariamente a Trastornos } \\
\text { neurocognitivos de la } \mathrm{CIE}-11\end{array}$ \\
\hline $\begin{array}{l}\text { Trastornos debidos al consumo de } \\
\text { sustancias no psicoactivas }\end{array}$ & $\begin{array}{l}\text { F55.x Abuso de sustancias que no producen } \\
\text { dependencia }\end{array}$ & \\
\hline
\end{tabular}


tamiento (juego o videojuego), la búsqueda de sustancias y su consumo a pesar de las consecuencias dañinas (Heinz et al., 2019). A pesar de que estos síntomas se han asociado con la liberación de dopamina en el estriado ventral, la liberación de dopamina del estriado ventral por sí sola no es un criterio suficiente de la propiedad adictiva de una droga. Por ejemplo, los reforzadores comunes como la comida y el sexo aumentan la transmisión de dopamina en el núcleo accumbens, pero a diferencia de las sustancias adictivas, su efecto está regulado por la previsibilidad de recompensa y la habituación. El rango actualizado y ampliado de 14 clases de sustancias refleja cambios en las sustancias cada vez más asociadas con las consecuencias para la salud pública en diferentes partes del mundo (ver Tabla 2).

abla 2. Comparación de sustancias psicoactivas de la CIE-10 y CIE-11

\begin{tabular}{ll}
\hline CIE-10 & CIE-11 \\
\hline Alcohol & Alcohol \\
Cannabinoides & Cannabis \\
& Cannabinoides sintéticos \\
Opioides & Opioides \\
Sedantes o hipnóticos & Sedantes, hipnóticos y ansiolíticos \\
Cocaína & Cocaína \\
Otros estimulantes, incluido & Estimulantes \\
cafeína & Catinonas sintéticos \\
& Cafeína \\
Alucinógenos & Alucinógenos \\
Tabaco & Nicotina \\
Disolventes volátiles & Disolventes volátiles \\
& MDMA o drogas relacionadas \\
& Drogas disociativas \\
\hline
\end{tabular}

Esto permitirá un seguimiento más preciso en los sistemas de salud y, por lo tanto, en datos de salud agregados como base para la formulación de respuestas clínicas, de salud pública y políticas sociales apropiadas a nivel nacional y global. Específicamente, las sustancias ansiolíticas se mencionan de forma explícita como parte de la clase de sustancias sedantes e hipnóticas. La cafeína se separa de otros estimulantes debido a la creciente importancia para la salud pública de ciertas formas de su uso como el uso de bebidas energéticas no están reguladas o mezclas de metanfetamina con cafeína. El tabaco de la CIE-10 se reemplaza por la nicotina en la CIE-11, lo que refleja el uso cada vez mayor de formas alternativas de nicotina, como por ejemplo a través de vaporizadores. Por otra parte, el MDMA o "éxtasis" y las drogas disociativas como la ketamina y la fenciclidina (PCP) se proponen como clases separadas de sustancias psicoactivas. En vista de la creciente importancia para la salud pública de las llamadas nuevas sustancias psicoactivas (Mounteney et al., 2016), se han propuesto cannabinoides sintéticos y catinonas sintéticas como nuevas clases de sustancias psicoactivas (Rhumorbarbe et al., 2019). De esta manera, el uso de cannabinoides sintéticos se puede analizar por separado del cannabis y la resina de cannabis (Mensen et al., 2019).

\section{Patrón de consumo perjudicial.}

Tal como se propuso para la CIE-10, el "uso perjudicial" en la CIE-11 se reemplaza por el "patrón de uso perjudicial”, que se puede especificar como continuo o episódico. Es importante destacar que el daño a la salud de los demás se ha incluido en la definición de uso nocivo. Esto refleja el creciente papel de este tipo de daño en la configuración de las políticas y las respuestas del programa, que es particularmente importante en relación con el consumo de tabaco. El daño a la salud de otros puede ser intencional, como en el caso de homicidios o violencia interpersonal durante la intoxicación con alcohol o drogas, o como resultado de la dificultad del usuario de la sustancia para desempeñar funciones sociales, profesionales o familiares, como en el caso de negligencia hacia los hijos. El daño a la salud de otros también se está evaluando para su posible uso en la mejora de la medición de la carga de la enfermedad causada por el uso de sustancias y los costos generales del uso de sustancias para las sociedades. Según lo propuesto para la CIE-11, un patrón de uso nocivo se define como uno que ha causado un daño clínicamente significativo a la salud física o mental de una persona o en el que el comportamiento inducido por sustancias ha causado un daño clínicamente significativo a la salud de otras personas. Una vez más, el daño puede ser causado por los efectos tóxicos de una sustancia, los efectos tóxicos directos o secundarios en los órganos y sistemas del cuerpo, o una vía de administración peligrosa.

\section{Un solo episodio de consumo nocivo.}

Se ha propuesto una nueva categoría de diagnóstico 'episodio único de uso nocivo' para la CIE-11, con el objetivo de facilitar el reconocimiento de episodios de uso de sustancias que causen daños a la salud en ausencia de las características de diagnóstico de dependencia de sustancias o una Patrón nocivo de uso de sustancias. Esta categoría está diseñada específicamente para su uso en el contexto de una amplia gama de servicios de salud, especialmente en centros de atención primaria y emergencias, más que en centros de tratamiento especializados para trastornos mentales o por uso de sustancias. La definición propuesta es equivalente a la definición de un patrón de uso dañino, excepto que el daño ha sido causado por un solo episodio de uso. La inclusión y expansión del concepto de uso nocivo como se propone para la CIE-11 son extremadamente importantes porque brinda oportunidades para la prevención, así como el reconocimiento temprano de conductas relevantes relacionadas con el uso de sustancias. Estas formas de proble- 
mas pueden ser preclínicas en comparación con la forma en que se encuentran los trastornos por uso de sustancias en entornos especializados. La identificación del uso nocivo, ya sea como un patrón o como un episodio único, también ayudará a identificar a las personas que puedan responder a intervenciones psicológicas breves realizadas por proveedores de atención médica no especializados. A través de la agregación de datos de encuentros de salud, estas categorías también pueden apoyar un mejor monitoreo del impacto del uso de sustancias en la salud de la población.

\section{Trastornos por comportamientos adictivos}

Razón para introducir trastornos de la categoría debido a un comportamiento adictivo con un trastorno de consumo de sustancias:

- Frecuente concurrencia con trastornos por consumo de sustancias más que con trastornos del control de los impulsos.

- Patrones de desarrollo e historia similar

- Comparten bases neurobiológicas y déficits cognitivos

- Están implicados los circuitos de recompensa dopaminérgicos hiposensibles

- Responden de manera parecida a los tratamientos conductuales y farmacológicos (por ejemplo, antagonistas opioides)

- Comparten similitudes genéticas

En el subapartado de comportamientos adictivos se incluyen dos categorías diagnósticas: Juego patológico y Trastorno por videojuego. Al igual que en el DSM-5, el Juego patológico se incluye en este capítulo, y se omite del F63 Trastornos de los hábitos e impulsos. Como novedad, se añade el trastorno por videojuego que comparte similitudes con el juego patológico y con los trastornos por consumo de sustancias. La inclusión del Trastorno por videojuego se debe en parte al reconocimiento de la prevalencia creciente de videojuego problemático como un asunto de salud pública, sobre todo en países como China y Japón. Particularmente en España, el juego constituye todo un fenómeno en ebullición con gran impacto económico y social y con unas necesidades regulatorias y de políticas preventivas específicas (Choliz y Saiz-Ruiz, 2016). La Dirección General de Ordenación del Juego (DGOJ) señala en el informe de 2017 que las máquinas recreativas con premio, loterías y apuestas por Internet (apuestas deportivas y póker) y los videojuegos (tanto fuera de línea como a través de internet) han sido las preferidas por los más jóvenes (18-35 años) mientras que los juegos problemáticos que con mayor frecuencia se han identificado en los grupos de más edad son las máquinas tragaperras, la lotería y las quinielas (Dirección General de Ordenación del Juego, 2017). Tanto el Juego patológico como el Trastorno de videojuego tienen dos especificadores: predominante en línea (online) o sin conexión (offline).

\section{Conclusiones}

La clasificación propuesta de trastornos por consumo de sustancias y comportamientos adictivos en la CIE-11 incluye una gama de categorías diagnósticas que cubren un amplio espectro de condiciones de salud. Éstos reflejan diferentes niveles y patrones de uso de sustancias que oscilan entre un consumo puntual nocivo hasta el comportamiento consolidado de la dependencia. Se han realizado cambios sustanciales basados en estudios sistemáticos y trabajos de campo para aumentar la validez científica sustentada en la evidencia actual y para mejorar la utilidad clínica y la aplicabilidad global.

Uno de los objetivos principales de este sistema clasificatorio es facilitar el reconocimiento temprano del impacto negativo del consumo de sustancias sobre la salud y la provisión de intervenciones de prevención y tratamiento dirigidas a los diferentes dispositivos de atención correspondientes. Otro de los propósitos de la undécima revisión pretende potenciar la capacidad de los estados miembros de la OMS para monitorizar las consecuencias para la salud del consumo de sustancias a nivel de la población en aras de aplicar estrategias y políticas mundiales de gran calado y para apoyar la planificación efectiva y el desarrollo de sistemas de tratamiento. Los conceptos y definiciones para los problemas relacionados con el consumo de sustancias que se han plasmado con un enfoque de salud pública y con la participación de un amplio espectro de profesionales de la salud resultarán más válidos y tendrán una gran repercusión en diferentes niveles del sistema de salud tanto para su identificación como para su gestión. Por otra parte consideramos que la CIE-11 constituirá una herramienta útil para reducir la brecha de tratamiento entre aquellos que pueden beneficiarse de las intervenciones de prevención y tratamiento, y aquellos que realmente los reciben y, con el tiempo, mejorar la cobertura de las diferentes intervenciones en el campo de las adicciones, incluyendo aquellas a nuevas sustancias psicoactivas y a los comportamientos recientemente incorporados, como el juego patológico y el trastorno de videojuego.

\section{Conflicto de intereses}

Los autores declaran que no existe ningún conflicto de interés con las propuestas terapéuticas que se mencionan en este editorial.

\section{Referencias}

Asociación Americana de Psiquiatría. (2014). Manual Diagnóstico y Estadístico de los Trastornos Mentales $5^{a}$ edición (DSM-5). Madrid: Editorial Médica Panamericana.

Ayuso Mateos, J. L. (2018). The process of preparing the chapter on mental and behavioural disorders of the ICD 11. Revista de Psiquiatría y Salud Mental, 11, 127-129. doi:10.1016/j.rpsm.2018.02.004. 
Choliz, M. y Saiz-Ruiz, J. (2016). Regulating gambling to prevent addiction: more necessary now than ever. Adicciones, 28, 174-181. doi:10.20882/adicciones.820.

Plan de acción de la UE en materia de lucha contra la droga 2017-2020 (2017/C 215/02) C.F.R. (2017).

Dirección General de Ordenación del Juego. (2017). Estudio y análisis de los factores de riesgo del trastorno de juego en población clínica española. Recuperado de https://www. ordenacionjuego.es/es/estudio-prevalencia.

Dolengevich-Segal, H., Rodriguez Salgado, B., Gomez-Arnau Ramirez, J. y Sanchez-Mateos, D. (2015). New Psychoactive Drugs. Adicciones, 27, 231-232.

Dolengevich-Segal, H., Rodriguez Salgado, B., Ballesteros-López, J. y Molina-Prado, R. (2017). Chemsex. An emergent phenomenon. Adicciones, 29, 207-209. doi:10.20882/adicciones.894.

Gonzalez-Marti, I., Fernandez-Bustos, J. G., Contreras Jordan, O. R. y Sokolova, M. (2018). Muscle dysmorphia: detection of the use-abuse of anabolic adrogenic steroids in a Spanish sample. Adicciones, 30, 243-250. doi:10.20882/adicciones.853.

Heinz, A., Daedelow, L. S., Wackerhagen, C. y Di Chiara, G. (2019). Addiction theory matters-Why there is no dependence on caffeine or antidepressant medication. Addiction Biology. Avance de publicación on-line. doi:10.1111/adb.12735.

Keeley, J. W., Gaebel, W., First, M. B., Peterson, D. L., Rebello, T., Sharan, P. y Reed, G. M. (2018). Psychotic disorder symptom rating scales: Are dichotomous or multi-point scales more clinically useful?-An ICD-11 field study. Schizophrenia Research, 202, 254-259. doi:10.1016/j.schres.2018.07.006.

Medina-Mora, M. E., Robles, R., Rebello, T. J., Dominguez, T., Martinez, N., Juarez, F., . . Reed, G. M. (2019). ICD11 guidelines for psychotic, mood, anxiety and stress-related disorders in Mexico: Clinical utility and reliability. International Journal of Clinical and Health Psychology, 19, 1-11. doi:10.1016/j.ijchp.2018.09.003.

Mensen, V. T., Vreeker, A., Nordgren, J., Atkinson, A., de la Torre, R., Farre, M., . . . Brunt, T. M. (2019). Psychopathological symptoms associated with synthetic cannabinoid use: a comparison with natural cannabis. Psychopharmacology (Berlin). Avance de publicación on-line. doi:10.1007/s00213-019-05238-8.

Mounteney, J., Griffiths, P., Sedefov, R., Noor, A., Vicente, J. y Simon, R. (2016). The drug situation in Europe: an overview of data available on illicit drugs and new psychoactive substances from European monitoring in 2015. Addiction, 111, 34-48. doi:10.1111/add.13056.

Organización Mundial de la Salud. (2013). Plan de acción sobre salud mental 2013-2020. Ginebra.

Organización Mundial de la Salud. (2019, (Versión : 04 / 2019)). CIE-11 para estadísticas de mortalidad y morbilidad. Recuperado de https://www.who.int/classifications/icd/en/.
Peterson, D. L., Webb, C. A., Keeley, J. W., Gaebel, W., Zielasek, J., Rebello, T. J., . . Reed, G. M. (2019). The reliability and clinical utility of ICD-11 schizoaffective disorder: A field trial. Schizophrizophrenia Research. Avance de publicación on-line. doi:10.1016/j.schres.2019.02.011.

Poznyak, V., Reed, G. M. y Medina-Mora, M. E. (2018). Aligning the ICD-11 classification of disorders due to substance use with global service needs. Epidemiology and Psychiatric Sciences, 27, 212-218. doi:10.1017/ S2045796017000622.

Reed, G. M. y Ayuso-Mateos, J. L. (2011). Towards a more clinically useful International World Health Organisation classification of mental disorders. Revista de Psiquiatría y Salud Mental, 4, 113-116. doi:10.1016/j. rpsm.2011.04.003.

Reed, G. M., First, M. B., Kogan, C. S., Hyman, S. E., Gureje, O., Gaebel, W., . . . Saxena, S. (2019). Innovations and changes in the ICD-11 classification of mental, behavioural and neurodevelopmental disorders. World Psychiatry, 18, 3-19. doi:10.1002/wps.20611.

Reed, G. M., Keeley, J. W., Rebello, T. J., First, M. B., Gureje, O., Ayuso-Mateos, J. L., . . . Medina-Mora, M. E. (2018). Clinical utility of ICD-11 diagnostic guidelines for high-burden mental disorders: results from mental health settings in 13 countries. World Psychiatry, 17, 306315. doi:10.1002/wps.20581.

Reed, G. M., Sharan, P., Rebello, T. J., Keeley, J. W., Elena Medina-Mora, M., Gureje, O., . . Pike, K. M. (2018). The ICD-11 developmental field study of reliability of diagnoses of high-burden mental disorders: results among adult patients in mental health settings of 13 countries. World Psychiatry, 17, 174-186. doi:10.1002/wps.20524.

Rhumorbarbe, D., Morelato, M., Staehli, L., Roux, C., Jaquet-Chiffelle, D. O., Rossy, Q. y Esseiva, P. (2019). Monitoring new psychoactive substances: Exploring the contribution of an online discussion forum. International Journal of Drug Policy. Avance de publicación on-line. doi:10.1016/j.drugpo.2019.03.025.

Robles Garcia, R. y Ayuso-Mateos, J. L. (2019). CIE-11 and the depathologisation of the transgender condition. Revista de Psiquiatría y Salud Mental, 12, 65-67. doi:10.1016/j.rpsm.2019.01.002.

Stein, D. J.y Reed, G. M. (2019). Global mental health and psychiatric nosology: DSM-5, ICD-11, and RDoC. Brazilian Journal of Psychiatry, 41, 2. doi:10.1590/1516-44462018-4101.

Wegman, M. P., Altice, F. L., Kaur, S., Rajandaran, V., Osornprasop, S., Wilson, D., . . Kamarulzaman, A. (2017). Relapse to opioid use in opioid-dependent individuals released from compulsory drug detention centres compared with those from voluntary methadone treatment centres in Malaysia: a two-arm, prospective observational study. Lancet Global Health, 5, e198-e207. doi:10.1016/ S2214-109X(16)30303-5. 